\title{
Nonexistence of Global Solutions to the Initial Boundary Value Problem for the Singularly Perturbed Sixth-Order Boussinesq-Type Equation
}

\author{
Changming Song, Jina Li, and Ran Gao \\ College of Science, Zhongyuan University of Technology, Zhengzhou 450007, China \\ Correspondence should be addressed to Changming Song; cmsongh@163.com
}

Received 9 March 2014; Accepted 10 May 2014; Published 26 May 2014

Academic Editor: Qiu-Ming Luo

Copyright (C) 2014 Changming Song et al. This is an open access article distributed under the Creative Commons Attribution License, which permits unrestricted use, distribution, and reproduction in any medium, provided the original work is properly cited.

We are concerned with the singularly perturbed Boussinesq-type equation including the singularly perturbed sixth-order Boussinesq equation, which describes the bidirectional propagation of small amplitude and long capillary-gravity waves on the surface of shallow water for bond number (surface tension parameter) less than but very close to 1/3. The nonexistence of global solution to the initial boundary value problem for the singularly perturbed Boussinesq-type equation is discussed and two examples are given.

\section{Introduction}

In the numerical study of the ill-posed Boussinesq equation,

$$
u_{t t}=u_{x x}+\left(u^{2}\right)_{x x}+u_{x x x x} .
$$

Darapi and Hua [1] proposed the singularly perturbed Boussinesq equation

$$
u_{t t}=u_{x x}+\left(u^{2}\right)_{x x}+u_{x x x x}+\delta u_{x x x x x x}
$$

as a dispersive regularization of the ill-posed classical Boussinesq equation (1), where $\delta>0$ is a small parameter. The authors use both filtering and regularization techniques to control growth of the errors and to provide better approximate solutions of this equation. Dash and Daripa [2] presented a formal derivation of (2) from two-dimensional potential flow equations for water waves through an asymptotic series expansion for small amplitude and long wave length. The physical relevance of (2) in the context of water waves was also addressed in [2]; it was shown that (2) actually describes the bidirectional propagation of small amplitude and long capillary-gravity waves on the surface of shallow water for bond number (surface tension parameter) less than but very close to $1 / 3$. On the basis of far-field analysis and heuristic arguments, Daripa and Dash [3] proved that the traveling wave solutions of (2) are weakly nonlocal solitary waves characterized by small amplitude fast oscillations in the far-field and obtained weakly nonlocal solitary wave solutions of (2). Feng [4] investigated the generalized Boussinesq equation including the singularly perturbed Boussinesq equation

$$
u_{t t}=[Q(u)]_{x x}+\sum_{i=1}^{n} b_{i} u_{(2 i+2) x},
$$

where $Q(u)=u+b_{0} u^{r}, u_{(2 i+2) x}=\left(\partial^{2 i+2} u\right) /\left(\partial x^{2 i+2}\right), r$ and $b_{i}(i=1,2, \ldots, n)$ are all real constants. It is easily seen that the choices $b_{0}=1, r=2, n=2, b_{1}=1$, and $b_{2}=\delta$ lead (3) to the singularly perturbed Boussinesq equation (2). By the means of two proper ansatzs, the author obtained explicit traveling solitary wave solutions of the generalized Boussinesq equation (3). To the best of our knowledge, however, there have not been any discussions on global solutions of the initial boundary value problem for (2) in the literature; recently, Song et al. [5] discussed the initial boundary value problem for the singularly perturbed Boussinesq-type equation

$$
u_{t t}=u_{x x}+\sigma(u)_{x x}+\alpha u_{x^{4}}+\beta u_{x^{6}}, \quad x \in \Omega, t>0,
$$


with the initial boundary value conditions

$$
\begin{gathered}
u_{x}(0, t)=u_{x}(1, t)=u_{x^{3}}(0, t)=u_{x^{3}}(1, t)=u_{x^{5}}(0, t) \\
=u_{x^{5}}(1, t)=0, \quad t>0, \\
u(x, 0)=u_{0}(x), \quad u_{t}(x, 0)=u_{1}(x), \quad x \in \bar{\Omega},
\end{gathered}
$$

or with

$$
\begin{gathered}
u(0, t)=u(1, t)=u_{x x}(0, t)=u_{x x}(1, t)=u_{x^{4}}(0, t) \\
=u_{x^{4}}(1, t)=0, \quad t>0, \\
u(x, 0)=u_{0}(x), \quad u_{t}(x, 0)=u_{1}(x), \quad x \in \bar{\Omega},
\end{gathered}
$$

where, and in the sequel $u_{x^{i}}=\partial^{i} u / \partial x^{i}, \sigma(s)$ is a given nonlinear function, $\alpha>0$ and $\beta>0$ are real numbers, $u_{0}(x)$ and $u_{1}(x)$ are given initial value functions, and $\Omega=(0,1)$. By virtue of the Galerkin method and prior estimates, under the assumption " $\sigma$ ' $(s)$ is bounded below and $\sigma(s)$ satisfies some smooth condition," the existence and uniqueness of the global generalized solution and the global classical solution of the initial boundary value problem (4), (5) and (4), (6) are proved, respectively. But if $\sigma^{\prime}(s)$ is not bounded below, does the above-mentioned problem have any global solution? In this paper, we employ the energy method and the Jensen inequality to prove that the global solutions of the initial boundary value problem (4), (5) and (4), (6) cease to exist in a finite time, respectively. At last, we show that the global solution of the initial boundary value problem (2), (6) blows up in a finite time.

The paper is organized as follows. In Section 2, the main results are stated. The nonexistence of global solution of problem (4), (5) and (4), (6) is discussed in Section 3. In Section 4 , we study the initial boundary problem (2), (6) and give two examples satisfying the theorems (Theorems 1-6).

\section{Main Theorems}

Throughout this paper, we use the abbreviations $\|\cdot\|=$ $\|\cdot\|_{L^{2}(\Omega)}$. In the following we state the main results of this paper, where the existence of Theorems 1-4 has been proved in $[5]$.

Theorem 1 (see [5]). Assume that $u_{0} \in H^{6}(\Omega), u_{1} \in H^{3}(\Omega)$, $\int_{0}^{1} u_{0}(x) d x=\int_{0}^{1} u_{1}(x) d x=0, u_{0 x^{2 k+1}}(0, t)=u_{0 x^{2 k+1}}(1, t)=$ $u_{1 x^{2 k+1}}(0, t)=u_{1 x^{2 k+1}}(1, t)=0(k=0,1,2), \sigma \in C^{5}(\mathbf{R})$, and $\sigma^{\prime}(s)$ is bounded below; namely, there exists a constant $C_{0}$ such that $\sigma^{\prime}(s) \geq C_{0}$, for any $s \in \mathbf{R}$. Then, for any $T>0$, the initial boundary value problem (4), (5) admits a unique global generalized solution $u(x, t)$ with

$$
\begin{gathered}
u \in C\left([0, T] ; H^{6}(\Omega)\right) \cap C^{1}\left([0, T] ; H^{3}(\Omega)\right) \\
\cap C^{2}\left([0, T] ; L^{2}(\Omega)\right) .
\end{gathered}
$$

Theorem 2 (see [5]). Assume that the assumptions of Theorem 1 hold, $u_{0} \in H^{10}(\Omega), u_{1} \in H^{7}(\Omega)$, and $\sigma \in C^{9}(\mathbf{R})$.
Then, the initial boundary value problem (4), (5) admits a unique global classical solution $u(x, t)$.

Theorem 3 (see [5]). Assume that $u_{0} \in H^{6}(\Omega), u_{1} \in H^{3}(\Omega)$, $u_{0 x^{2 k}}(0, t)=u_{0 x^{2 k}}(1, t)=u_{1 x^{2 k}}(0, t)=u_{1 x^{2 k}}(1, t)=0(k=$ $0,1,2), \sigma \in C^{5}(\mathbf{R}), \sigma^{(2 i)}(0)=0(i=1,2)$, and $\sigma^{\prime}(s)$ is bounded below. Then, for any $T>0$, the initial boundary value problem (4), (6) admits a unique global generalized solution $u(x, t)$ with

$$
\begin{gathered}
u \in C\left([0, T] ; H^{6}(\Omega)\right) \cap C^{1}\left([0, T] ; H^{3}(\Omega)\right) \\
\cap C^{2}\left([0, T] ; L^{2}(\Omega)\right) .
\end{gathered}
$$

Theorem 4 (see [5]). Assume that the assumptions of Theorem 3 hold, $u_{0} \in H^{10}(\Omega), u_{1} \in H^{7}(\Omega), \sigma \in C^{9}(\mathbf{R})$, and $\sigma^{(2 i)}(0)=0(i=3,4)$. Then, the initial boundary value problem (4), (6) admits a unique global classical solution $u(x, t)$.

Theorem 5. Assume that (1) $\sigma(s) s \leq \mu \Gamma(s), \Gamma(s) \leq-\gamma|s|^{m+1}$, where $\Gamma(s)=\int_{0}^{s} \sigma(\tau) d \tau, \mu>2, \gamma>0$, and $m>1$ are constants, and (2) $u_{0} \in H^{2}, u_{1} \in L^{2}, \int_{0}^{1} u_{0}(x) d x=\int_{0}^{1} u_{1}(x) d x=0$, and

$$
\begin{aligned}
E_{0}= & \int_{0}^{1}\left(\int_{0}^{x} u_{1}(\xi) d \xi\right)^{2} d x+\left\|u_{0}\right\|^{2}-\alpha\left\|u_{0}^{\prime}\right\|^{2}+\beta\left\|u_{0}^{\prime \prime}\right\|^{2} \\
& +2 \int_{0}^{1} \int_{0}^{u_{0}} \sigma(s) d s d x \leq-\left[\frac{2}{D\left(1-e^{(1-m) / 4}\right)^{2}}\right]^{2 /(m-1)},
\end{aligned}
$$

where $D=\gamma(\mu-2) /\left[2^{(m-7) / 2}(m+3)\right]$. Then the solution $u(x, t)$ of initial boundary value problem (4), (5) blows up in a finite time $T_{0}$; namely,

$$
\|u(t)\|_{L^{1}(\Omega)}^{2}+\int_{0}^{t}\|u(\tau)\|^{2} d \tau \longrightarrow+\infty \quad \text { as } t \longrightarrow T_{0}^{-},
$$

where $T_{0}$ is defined in the proof.

Theorem 6. Assume that (1) $\sigma \in C^{2}(\mathbf{R}), \sigma(0)=0$, and one of the following conditions holds: (i) $\sigma(s)$ is a convex and even function, $\sigma(s) \geq a s^{m}$, where $a>0$ and $m>$ 1 are real numbers, (ii) $\sigma(s)$ is a convex function, $\sigma(s) \geq$ $a s^{m}$, where $a>0$ is a real number and $m \geq 2$ is an even number, and (2) $-(\pi / 2) \int_{0}^{1} u_{0}(x) \sin \pi x d x>\max \{0$, $\left.\left(\left(\beta \pi^{4}-\alpha \pi^{2}+1\right) / a\right)^{1 /(m-1)}\right\},-(\pi / 2) \int_{0}^{1} u_{1}(x) \sin \pi x d x>0$. Then the solution $u(x, t)$ of the initial boundary value problem (4), (6) blows up in a finite time $T_{1}$; namely,

$$
\|u(t)\| \longrightarrow+\infty, \quad \text { as } t \longrightarrow T_{1}^{-},
$$

where $T_{1}$ is defined in the proof.

\section{Nonexistence of Global Solutions of Problem (4), (5) and (4), (6)}

We first quote the following lemmas. 
Lemma 7 (see Li [6]). Assume that $\dot{u}=G(t, u), \dot{v} \geq G(t, v)$, $G \in C([0,+\infty) \times \mathbf{R}), t_{0} \leq t<+\infty$, and $u\left(t_{0}\right)=v\left(t_{0}\right)$. Then $u(t) \geq v(t)$ as $t \geq t_{0}$.

Lemma 8 (Jensen inequality [7]). Assume that $\varphi(u): u \in$ $[\alpha, \beta] \mapsto \mathbf{R}$ is a convex function, $f: x \in \Omega \mapsto[\alpha, \beta]$, and $P(x)$ is a continuous function, $P(x) \geq 0, P(x) \neq 0$. Then

$$
\varphi\left(\frac{\int_{\Omega} f(x) P(x) d x}{\int_{\Omega} P(x) d x}\right) \leq \frac{\int_{\Omega} \varphi(f(x)) P(x) d x}{\int_{\Omega} P(x) d x} .
$$

Integrating both sides of (4) over $(0,1)$ and using (5) and the assumption of Theorem 1 , we obtain $\int_{0}^{1} u(x, t) d x=0, t \geq 0$. Let $v(x, t)=\int_{0}^{x} u(\xi, t) d \xi$; then $u=v_{x}$ and $v$ satisfies

$$
\begin{gathered}
v_{t t}=v_{x x}+\sigma\left(v_{x}\right)_{x}+\alpha v_{x^{4}}+\beta v_{x^{6}}, \quad x \in \Omega, t>0, \\
\begin{aligned}
& v(0, t)= v(1, t)=v_{x x}(0, t)=v_{x x}(1, t)=v_{x^{4}}(0, t) \\
& \quad=v_{x^{4}}(1, t)=0, \quad t>0, \\
& v(x, 0)=v_{0}(x), \quad v_{t}(x, 0)=v_{1}(x), \quad x \in \bar{\Omega},
\end{aligned}
\end{gathered}
$$

where $v_{0}(x)=\int_{0}^{x} u_{0}(\xi) d \xi$ and $v_{1}(x)=\int_{0}^{x} u_{1}(\xi) d \xi$.

Proof of Theorem 5. Multiplying both sides of (13) by $2 v_{t}$, integrating by parts, and using condition (2) of Theorem 5 , we have

$$
\dot{E}(t)=0, \quad E(t)=E(0)=E_{0}<0, \quad t>0,
$$

where

$$
\begin{aligned}
E(t)= & \left\|v_{t}(t)\right\|^{2}+\left\|v_{x}(t)\right\|^{2}-\alpha\left\|v_{x x}(t)\right\|^{2}+\beta\left\|v_{x^{3}}(t)\right\|^{2} \\
& +2 \int_{0}^{1} \Gamma\left(v_{x}(x, t)\right) d x .
\end{aligned}
$$

Let

$$
F(t)=\|v(t)\|^{2}+\int_{0}^{t} \int_{0}^{\tau}\left\|v_{x}(s)\right\|^{2} d s d \tau .
$$

By virtue of condition (1) of Theorem 5 and noting that

$$
\begin{aligned}
\mu \int_{0}^{1} \Gamma\left(v_{x}(x, t)\right) d x= & E_{0}-\left\|v_{t}(t)\right\|^{2}-\left\|v_{x}(t)\right\|^{2}+\alpha\left\|v_{x x}(t)\right\|^{2} \\
& -\beta\left\|v_{x^{3}}(t)\right\|^{2}+(\mu-2) \int_{0}^{1} \Gamma\left(v_{x}\right) d x,
\end{aligned}
$$

we obtain

$$
\begin{aligned}
\ddot{F}(t)= & 2 \int_{0}^{1} v v_{t t} d x+2\left\|v_{t}(t)\right\|^{2}+\left\|v_{x}(t)\right\|^{2} \\
= & -2 \int_{0}^{1}\left(v_{x}^{2}-\alpha v_{x x}^{2}+\beta v_{x^{3}}^{2}+\sigma\left(v_{x}\right) v_{x}\right) d x \\
& +2\left\|v_{t}(t)\right\|^{2}+\left\|v_{x}(t)\right\|^{2} \\
\geq & -2 E_{0}+\left\|v_{x}(t)\right\|^{2}+4\left\|v_{t}(t)\right\|^{2}-2(\mu-2) \int_{0}^{1} \Gamma\left(v_{x}\right) d x \\
\geq & -2 E_{0}+2 \gamma(\mu-2) \int_{0}^{1}\left|v_{x}\right|^{m+1} d x .
\end{aligned}
$$

It follows from (20) that

$$
\begin{aligned}
\dot{F}(t) \geq- & -2 E_{0} t+2 \gamma(\mu-2) \int_{0}^{t} \int_{0}^{1}\left|v_{x}(x, \tau)\right|^{m+1} d x d \tau+\dot{F}(0), \\
F(t) \geq & -E_{0} t^{2}+2 \gamma(\mu-2) \int_{0}^{t} \int_{0}^{\tau} \int_{0}^{1}\left|v_{x}(x, s)\right|^{m+1} d x d s d \tau \\
& +\dot{F}(0) t+F(0),
\end{aligned}
$$

where $\dot{F}(0)=2 \int_{0}^{1}\left(\int_{0}^{x} u_{0}(\xi) d \xi \int_{0}^{x} u_{1}(\xi) d \xi\right) d x$ and $F(0)=$ $\left\|\int_{0}^{x} u_{0}(\xi) d \xi\right\|^{2}$. Combining (20) with (22) leads to

$$
\begin{aligned}
\ddot{F}(t) & +F(t) \\
\geq & -E_{0} t^{2}+\dot{F}(0) t+F(0)-2 E_{0} \\
& +2 \gamma(\mu-2)\left(\int_{0}^{1}\left|v_{x}\right|^{m+1} d x\right. \\
& \left.+\int_{0}^{t} \int_{0}^{\tau} \int_{0}^{1}\left|v_{x}(x, s)\right|^{m+1} d x d s d \tau\right) .
\end{aligned}
$$

Making use of the Hölder inequality, we get

$$
\begin{gathered}
\int_{0}^{1}\left|v_{x}\right|^{m+1} d x \geq\left\|v_{x}(t)\right\|^{m+1} \\
\int_{0}^{t} \int_{0}^{\tau} \int_{0}^{1}\left|v_{x}(x, s)\right|^{m+1} d x d s d \tau \\
\geq\left(\frac{t^{2}}{2}\right)^{(1-m) / 2}\left(\int_{0}^{t} \int_{0}^{\tau} \int_{0}^{1}\left|v_{x}(x, s)\right|^{2} d x d s d \tau\right)^{(1+m) / 2}
\end{gathered}
$$


Substituting (24) into (23) and using the Poincaré inequality $\left(\left\|v_{x}(t)\right\| \geq\|v(t)\|\right)$ and the inequality $a^{n}+b^{n} \geq 2^{1-n}(a+b)^{n}$ $(a, b \geq 0, n \geq 1)$, we conclude that when $t \geq 1$,

$$
\begin{aligned}
& \ddot{F}(t)+F(t) \\
& \geq 2 \gamma(\mu-2) t^{1-m} \\
& \quad \times\left[\left(\left\|v_{x}(t)\right\|^{2}\right)^{(m+1) / 2}\right. \\
& \left.\quad+\left(\int_{0}^{t} \int_{0}^{\tau} \int_{0}^{1}\left|v_{x}(x, s)\right|^{2} d x d s d \tau\right)^{(m+1) / 2}\right] \\
& \quad-E_{0} t^{2}+\dot{F}(0) t+F(0)-2 E_{0} \\
& \geq 2^{(3-m) / 2} \gamma(\mu-2) t^{1-m} F^{(1+m) / 2}(t) \\
& \quad-E_{0} t^{2}+\dot{F}(0) t+F(0)-2 E_{0} .
\end{aligned}
$$

Choose $t_{0} \geq 1$ such that

$$
-2 E_{0} t_{0}+\dot{F}(0) \geq 0, \quad-E_{0} t_{0}^{2}+\dot{F}(0) t_{0}+F(0)-2 E_{0} \geq 0,
$$

and thus (21) and (22) imply that $\dot{F}(t) \geq 0$ and $F(t) \geq 0$, as $t \geq t_{0}$. Multiplying both sides of (25) by $2 \dot{F}(t)$, we have

$$
\frac{d}{d t}\left[\dot{F}^{2}(t)+F^{2}(t)\right] \geq D t^{1-m} \frac{d}{d t} F^{(m+3) / 2}(t)+H(t), \quad t \geq t_{0},
$$

where

$$
\begin{gathered}
D=\frac{\gamma(\mu-2)}{2^{(m-7) / 2}(m+3)}, \\
H(t)=2 \dot{F}(t)\left(-E_{0} t^{2}+\dot{F}(0) t+F(0)-2 E_{0}\right) .
\end{gathered}
$$

Equation (27) implies that

$$
\begin{array}{r}
\frac{d}{d t}\left[t^{m-1}\left(\dot{F}^{2}(t)+F^{2}(t)\right)-D F^{(m+3) / 2}(t)\right] \geq t^{m-1} H(t), \\
t \geq t_{0} .
\end{array}
$$

Since

$$
\begin{aligned}
& \int_{t_{0}}^{t} \tau^{m-1} H(\tau) d \tau \\
& \geq \int_{t_{0}}^{t} 2\left(-2 E_{0} \tau+\dot{F}(0)\right)\left(-E_{0} \tau^{2}+\dot{F}(0) \tau+F(0)\right. \\
&\left.-2 E_{0}\right) d \tau \longrightarrow+\infty, \quad t \longrightarrow+\infty,
\end{aligned}
$$

there exists a $t_{1}>t_{0}$ such that

$$
\begin{gathered}
\int_{t_{0}}^{t} \tau^{m-1} H(\tau) d \tau+t_{0}^{m-1}\left(\dot{F}^{2}\left(t_{0}\right)+F^{2}\left(t_{0}\right)\right) \\
-D F^{(m+3) / 2}\left(t_{0}\right) \geq 0, \quad t>t_{1} .
\end{gathered}
$$

By (31), integrating both sides of (29) over $\left(t_{0}, t\right)$, we obtain

$$
t^{m-1}\left[\dot{F}^{2}(t)+F^{2}(t)\right] \geq D F^{(m+3) / 2}(t), \quad t \geq t_{1} .
$$

Namely,

$$
\dot{F}(t)+F(t) \geq \sqrt{2 D} t^{(1-m) / 2} F^{(m+3) / 4}(t), \quad t \geq t_{1} .
$$

In order to use Lemma 7, we consider the following initial value problem of the Bernoulli equation:

$$
\begin{gathered}
\dot{X}+X=\sqrt{2 D} t^{(1-m) / 2} X^{(m+3) / 4}, \quad t>t_{1}, \\
X\left(t_{1}\right)=F\left(t_{1}\right) .
\end{gathered}
$$

We can obtain the solution of the initial value problem (34) as follows:

$$
X(t)=e^{-\left(t-t_{1}\right)}\left[F^{(1-m) / 4}\left(t_{1}\right)-\frac{m-1}{4} \sqrt{2 D}\right.
$$

$$
\begin{gathered}
\left.\times \int_{t_{1}}^{t} \tau^{(1-m) / 2} e^{((1-m) / 4)\left(\tau-t_{1}\right)} d \tau\right]^{4 /(1-m)} \\
=e^{-\left(t-t_{1}\right)} F\left(t_{1}\right) I^{4 /(1-m)}(t), \quad t \geq t_{1}
\end{gathered}
$$

where

$$
\begin{aligned}
I(t)= & 1-\frac{m-1}{4} \sqrt{2 D} F^{(m-1) / 4}\left(t_{1}\right) \\
& \times \int_{t_{1}}^{t} \tau^{(1-m) / 2} e^{((1-m) / 4)\left(\tau-t_{1}\right)} d \tau .
\end{aligned}
$$

By (36), we know that $I\left(t_{1}\right)=1>0$ and

$$
\begin{aligned}
J(t)= & \frac{m-1}{4} \sqrt{2 D} F^{(m-1) / 4}\left(t_{1}\right) \int_{t_{1}}^{t} \tau^{(1-m) / 2} e^{((1-m) / 4)\left(\tau-t_{1}\right)} d \tau \\
\geq & \frac{m-1}{4} \sqrt{2 D} F^{(m-1) / 4}\left(t_{1}\right)\left(1+t_{1}\right)^{(1-m) / 2} \\
& \times \int_{t_{1}}^{t_{1}+1} e^{((1-m) / 4)\left(\tau-t_{1}\right)} d \tau \\
= & \sqrt{2 D} F^{(m-1) / 4}\left(t_{1}\right)\left(1+t_{1}\right)^{(1-m) / 2} \\
& \times\left(1-e^{-(m-1) / 4}\right), \quad t \geq t_{1}+1 .
\end{aligned}
$$

It follows from (22) that

$$
\begin{aligned}
& F^{(m-1) / 4}(t)(1+t)^{(1-m) / 2} \\
& \quad \geq\left[\frac{-E_{0} t^{2}+\dot{F}(0) t+F(0)}{(t+1)^{2}}\right]^{(m-1) / 4} \longrightarrow\left(-E_{0}\right)^{(m-1) / 4}, \\
& t \longrightarrow+\infty .
\end{aligned}
$$


Choose $t_{1}$ sufficiently large such that $F^{(m-1) / 4}\left(t_{1}\right)(1+$ $\left.t_{1}\right)^{(1-m) / 2} \geq\left(-E_{0}\right)^{(m-1) / 4} / 2$. Combining (37) with (9), we obtain

$$
\begin{array}{r}
J(t) \geq \frac{1}{2} \sqrt{2 D}\left(-E_{0}\right)^{(m-1) / 4}\left(1-e^{(1-m) / 4}\right) \geq 1, \\
t \geq t_{1}+1 .
\end{array}
$$

Hence

$$
I(t)=1-J(t) \leq 0, \quad t \geq t_{1}+1 .
$$

By using the continuity of $I(t)$, there exists a finite time $T_{0}$, $t_{1}<T_{0} \leq t_{1}+1$ such that $I\left(T_{0}\right)=0$. Therefore, $X(t) \rightarrow+\infty$ as $t \rightarrow T_{0}^{-}$. By virtue of Lemma 7 , we deduce that $F(t) \geq$ $X(t), t \geq t_{1}$. Hence

$$
\begin{aligned}
F(t)= & \int_{0}^{1}\left(\int_{0}^{x} u(\xi, t) d \xi\right)^{2} d x \\
& +\int_{0}^{t} \int_{0}^{\tau} \int_{0}^{1} u^{2}(\xi, s) d \xi d s d \tau \longrightarrow+\infty
\end{aligned}
$$

as $t \rightarrow T_{0}^{-}$. It follows from (41) that

$$
F(t) \leq\left(\int_{0}^{1}|u(\xi, t)| d \xi\right)^{2}+t \int_{0}^{t}\|u(\tau)\|^{2} d \tau .
$$

Therefore,

$$
\|u(t)\|_{L^{1}(\Omega)}^{2}+\int_{0}^{t}\|u(\tau)\|^{2} d \tau \longrightarrow+\infty \quad \text { as } t \longrightarrow T_{0}^{-} .
$$

Theorem 5 is proved.

Proof of Theorem 6. Let

$$
y(t)=-\frac{\pi}{2} \int_{0}^{1} u(x, t) \sin \pi x d x .
$$

Multiplying both sides of (4) by $(\pi / 2) \sin \pi x$, integrating by parts over $[0,1]$, and making use of the Jensen inequality and condition (1) of Theorem 6, we have

$$
\begin{aligned}
\ddot{y}+\left(\pi^{2}-\alpha \pi^{4}+\beta \pi^{6}\right) y & =\frac{\pi^{3}}{2} \int_{0}^{1} \sigma(u) \sin \pi x d x \\
& \geq \pi^{2} \sigma\left(\frac{\pi}{2} \int_{0}^{1} u(x, t) \sin \pi x d x\right) \\
& \geq a \pi^{2} y^{m}, \quad t>0,
\end{aligned}
$$

and, from (6) and condition (2) of Theorem 6, we get

$$
\begin{aligned}
& y(0)=-\frac{\pi}{2} \int_{0}^{1} u_{0}(x) \sin \pi x d x=y_{0}>0, \\
& \dot{y}(0)=-\frac{\pi}{2} \int_{0}^{1} u_{1}(x) \sin \pi x d x=y_{1}>0 .
\end{aligned}
$$

Thus, we claim that

$$
y(t)>0, \quad \dot{y}(t)>0, \quad t>0 .
$$

In fact, if it is not true, then there exists a $t^{*}$ such that $\dot{y}(t)>0, t \in\left[0, t^{*}\right)$ and $\dot{y}\left(t^{*}\right)=0$. Then $y(t)$ is monotonically increasing on $\left[0, t^{*}\right]$; that is, $y(t) \geq y_{0}, t \in\left[0, t^{*}\right]$. By using (45) and condition (2) of Theorem 6 , we obtain

$$
\begin{aligned}
\ddot{y}(t) & \geq \pi^{2} y\left(a y^{m-1}-\beta \pi^{4}+\alpha \pi^{2}-1\right) \\
& >\pi^{2} y_{0}\left(a y_{0}^{m-1}-\beta \pi^{4}+\alpha \pi^{2}-1\right)>0, \quad\left(0, t^{*}\right],
\end{aligned}
$$

and hence $\dot{y}(t)$ is monotonically increasing on $\left[0, t^{*}\right]$, which contradicts the assumption $y\left(t^{*}\right)=0$. So claim (47) is valid.

Multiplying both sides of (45) by $2 \dot{y}$ and integrating the product over $[0, t]$ lead to

$$
\begin{aligned}
\dot{y}^{2} \geq & \frac{2 a \pi^{2}}{m+1}\left(y^{m+1}-y_{0}^{m+1}\right) \\
& -\left(\pi^{2}-\alpha \pi^{4}+\beta \pi^{6}\right)\left(y^{2}-y_{0}^{2}\right)+y_{1}^{2}=G(y) .
\end{aligned}
$$

Since $G\left(y_{0}\right)=y_{1}^{2}>0$ and

$$
\begin{aligned}
G^{\prime}(y) & =2 \pi^{2} y\left[a y^{m-1}-\left(1-\alpha \pi^{2}+\beta \pi^{4}\right)\right] \\
& \geq 2 \pi^{2} y_{0}\left[a y_{0}^{m-1}-\left(1-\alpha \pi^{2}+\beta \pi^{4}\right)\right] \geq 0,
\end{aligned}
$$

$G(y)>G\left(y_{0}\right)>0, t>0$. It follows from (49) that

$$
\dot{y} \geq\left[\frac{2 a \pi^{2}}{m+1}\left(y^{m+1}-y_{0}^{m+1}\right)\right.
$$

$$
\left.-\left(\pi^{2}-\alpha \pi^{4}+\beta \pi^{6}\right)\left(y^{2}-y_{0}^{2}\right)+y_{1}^{2}\right]^{1 / 2}, \quad t>0,
$$

and (51) implies that the interval $\left[0, T_{1}\right)$ of the existence of $y(t)$ is finite; namely,

$$
\begin{aligned}
T_{1} \leq \int_{y_{0}}^{+\infty} & {\left[\frac{2 a \pi^{2}}{m+1}\left(y^{m+1}-y_{0}^{m+1}\right)\right.} \\
& \left.\quad-\left(\pi^{2}-\alpha \pi^{4}+\beta \pi^{6}\right)\left(y^{2}-y_{0}^{2}\right)+y_{1}^{2}\right]^{-1 / 2} d y \\
<+\infty, &
\end{aligned}
$$

and $y(t) \rightarrow+\infty$ as $t \rightarrow T_{1}^{-}$; that is, $\int_{0}^{1} u(x, t) \sin \pi x d x \rightarrow$ $-\infty$ as $t \rightarrow T_{1}^{-}$. By the Hölder inequality, we have $\|u(t)\| \rightarrow$ $+\infty$, as $t \rightarrow T_{1}^{-}$. Theorem 6 is proved.

\section{Initial Boundary Value Problem (2), (6) and Some Examples}

By virtue of the Galerkin method [8] we can prove that initial boundary value problem (2), (6) admits a unique local 
generalized solution and a unique local classical solution. Moreover, by using Theorem 6, we obtain the following theorem.

Theorem 9. Assume that $u(x, t)$ is the generalized solution of initial boundary value problem (2), (6) and the following condition holds:

$$
\begin{aligned}
& y_{0}=-\frac{\pi}{2} \int_{0}^{1} u_{0}(x) \sin \pi x d x>\max \left\{0,\left(\delta \pi^{4}-\pi^{2}+1\right)\right\}, \\
& y_{1}=-\frac{\pi}{2} \int_{0}^{1} u_{1}(x) \sin \pi x d x>0 .
\end{aligned}
$$

Then

$$
\|u(t)\| \longrightarrow+\infty, \quad \text { as } t \longrightarrow T_{2}^{-}
$$

where

$$
\begin{aligned}
T_{2} \leq \int_{y_{0}}^{+\infty}[ & \frac{2 \pi^{2}}{3}\left(y^{3}-y_{0}^{3}\right) \\
& \left.-\left(\pi^{2}-\pi^{4}+\delta \pi^{6}\right)\left(y^{2}-y_{0}^{2}\right)+y_{1}^{2}\right]^{-1 / 2} d y<+\infty .
\end{aligned}
$$

Proof. A simple verification shows that all conditions of Theorem 6 are satisfied and thus Theorem 9 is proved immediately.

Example 1. We consider the following equation:

$$
u_{t t}=u_{x x}+a\left(|u|^{m-1} u\right)_{x x}+\alpha u_{x^{4}}+\beta u_{x^{6}}, \quad x \in \Omega, t>0
$$

with the initial boundary value conditions

$$
\begin{gathered}
u_{x}(0, t)=u_{x}(1, t)=u_{x^{3}}(0, t)=u_{x^{3}}(1, t)=u_{x^{5}}(0, t) \\
=u_{x^{5}}(1, t)=0, \quad t>0, \\
u(x, 0)=u_{0}(x), \quad u_{t}(x, 0)=u_{1}(x), \quad x \in \bar{\Omega},
\end{gathered}
$$

or with

$$
\begin{gathered}
u(0, t)=u(1, t)=u_{x x}(0, t)=u_{x x}(1, t)=u_{x^{4}}(0, t) \\
=u_{x^{4}}(1, t)=0, \quad t>0, \\
u(x, 0)=u_{0}(x), \quad u_{t}(x, 0)=u_{1}(x), \quad x \in \bar{\Omega},
\end{gathered}
$$

where $a \neq 0$ and $m>1$ are all real numbers, $u_{0}(x)=u_{1}(x)=$ $K_{0} \cos \pi x$, and $K_{0}>0$ is a constant.

(1) If $a>0$ and $m \geq 9$, a simple calculation shows that $\sigma\left(=a|s|^{m-1} s\right) \in C^{9}(\mathbf{R}), \sigma^{(2 i)}(0)=0(i=1,2,3,4)$, and $\sigma^{\prime}(s)=$ $a m|s|^{m-1} \geq 0$; that is, $\sigma^{\prime}(s)$ is bounded below. And $u_{0}(x)$ and $u_{1}(x)$ satisfy the conditions of Theorems 2 and 4, respectively; then by Theorems 2 and 4 we know that the initial boundary value problem (56), (57) and (56), (58) admits a unique global classical solution, respectively.

(2) If $a<0$ and $m>1$, we have $\sigma(s) s=a|s|^{m-1}, \Gamma(s)=$ $a|s|^{m-1} /(m+1)$; taking $\mu=m+1>2$ and $\gamma=-a /(m+1)$, then $\sigma(s) s=\mu \Gamma(s), \Gamma(s)=-\gamma|s|^{m-1} ;$ obviously, $u_{0} \in H^{2}, u_{1} \in L^{2}$, $\int_{0}^{1} u_{0}(x) d x=\int_{0}^{1} u_{1}(x) d x=0$, and

$$
\begin{aligned}
E_{0}= & \int_{0}^{1}\left(\int_{0}^{x} u_{1}(\xi) d \xi\right)^{2} d x+\left\|u_{0}\right\|^{2}-\alpha\left\|u_{0}^{\prime}\right\|^{2} \\
& +\beta\left\|u_{0}^{\prime \prime}\right\|^{2}+\frac{2 a}{m+1}\left\|u_{0}\right\|_{L^{m+1}(\Omega)}^{m+1} \\
= & \left(\frac{1}{\pi^{2}}+1-\alpha \pi^{2}+\beta \pi^{4}\right) \frac{K_{0}^{2}}{2} \\
& +\frac{2 a K_{0}^{m+1}}{m+1} \int_{0}^{1}|\cos \pi x|^{m+1} d x .
\end{aligned}
$$

We can take $K_{0}$ suitable large such that

$$
E_{0} \leq-\left[\frac{2}{D\left(1-e^{(1-m) / 4}\right)^{2}}\right]^{2 /(m-1)},
$$

where $D=-a(m-1) /\left[2^{(m-7) / 2}(m+1)(m+3)\right]$. Thus, all assumptions of Theorem 5 are satisfied; then by Theorem 5 we conclude that the solution of initial boundary value problem (56), (57) must blow up in a finite time $T_{0}$; namely,

$$
\|u(t)\|_{L^{1}(\Omega)}+\int_{0}^{t}\|u(\tau)\|^{2} d \tau \longrightarrow+\infty \quad \text { as } t \longrightarrow T_{0}^{-} .
$$

Example 2. We consider the following equation:

$$
u_{t t}=u_{x x}+a\left(u^{m}\right)_{x x}+\alpha u_{x^{4}}+\beta u_{x^{6}}, \quad x \in \Omega, t>0,
$$

with the initial boundary value conditions

$$
\begin{aligned}
u_{x}(0, t) & =u_{x}(1, t)=u_{x^{3}}(0, t)=u_{x^{3}}(1, t)=u_{x^{5}}(0, t) \\
& =u_{x^{5}}(1, t)=0, \quad t>0 \\
u(x, 0) & =u_{0}(x), \quad u_{t}(x, 0)=u_{1}(x), \quad x \in \bar{\Omega}
\end{aligned}
$$

or with

$$
\begin{gathered}
u(0, t)=u(1, t)=u_{x x}(0, t)=u_{x x}(1, t)=u_{x^{4}}(0, t) \\
=u_{x^{4}}(1, t)=0, \quad t>0, \\
u(x, 0)=u_{0}(x), \quad u_{t}(x, 0)=u_{1}(x), \quad x \in \bar{\Omega},
\end{gathered}
$$

where $a>0$ is a real number and $m>1$ is a positive integer, $u_{0}(x)=u_{1}(x)=-K_{1}$, and $K_{1}>0$ is a constant.

(1) If $a>0$ and $m$ is an odd number, a simple verification shows that all conditions of Theorems 2 and 4 are satisfied; then by Theorems 2 and 4 we know that the initial boundary value problem (62), (63) and (62), (64) admits a unique global classical solution, respectively. 
(2) If $a>0$ and $m$ is an even number, then $\sigma(s)\left(=a s^{m}\right)$ is a convex and even function, and we can take $K_{1}$ suitable large such that

$$
\begin{aligned}
-\frac{\pi}{2} \int_{0}^{1} u_{0}(x) \sin \pi x d x & \\
= & K_{1}>\max \left\{0,\left(\frac{\beta \pi^{4}-\alpha \pi^{2}+1}{a}\right)^{1 /(m-1)}\right\}, \\
& -\frac{\pi}{2} \int_{0}^{1} u_{1}(x) \sin \pi x d x=K_{1}>0 .
\end{aligned}
$$

Thus, by Theorem 6 , we deduce that the solution of initial boundary value problem (62), (64) must blow up in a finite time $T_{1}$; namely,

$$
\|u(t)\| \longrightarrow+\infty, \quad \text { as } t \longrightarrow T_{1}^{-} .
$$

\section{Conflict of Interests}

The authors declare that there is no conflict of interests regarding the publication of this paper.

\section{Acknowledgments}

This work is supported by the Natural Science Foundation of Henan Province of China (No. 122300410166 and No. 102300410275), the Natural Science Foundation of China (No. 11271336 and No. 11326167), and the Foundation of Henan Educational Committee (No. 13A110119).

\section{References}

[1] P. Darapi and W. Hua, "A numerical method for solving an ill-posed Boussinesq equation arising in water waves and nonlinear lattices," Applied Mathematics and Computation, vol. 101, no. 2, pp. 159-207, 1999.

[2] R. K. Dash and P. Daripa, "Analytical and numerical studies of a singularly perturbed Boussinesq equation," Applied Mathematics and Computation, vol. 126, no. 1, pp. 1-30, 2002.

[3] P. Daripa and R. K. Dash, "Weakly non-local solitary wave solutions of a singularly perturbed Boussinesq equation," Mathematics and Computers in Simulation, vol. 55, no. 4-6, pp. 393$405,2001$.

[4] Z. Feng, "Traveling solitary wave solutions to the generalized Boussinesq equation," Wave Motion, vol. 37, no. 1, pp. 17-23, 2003.

[5] C. Song, H. Li, and J. Li, "Initial boundary value problem for the singularly perturbed Boussinesq-type equation," Discrete and Continuous Dynamical Systems, vol. 2013, pp. 709-717, 2013.

[6] Y. Li, "Basic inequalities and the uniqueness of the solutions for differential equation (I)," Acta Scientiarum Naturalium Universitatis Jilinensis, vol. 1, pp. 7-22, 1960.

[7] E. F. Beckenbach and R. Bellman, Inequalities, Springer, 1961.

[8] J.-L. Lions, Quelques Méthodes de Résolution des Problèmes aux Limites non Linéaires, Dunod; Gauthier-Villars, Paris, France, 1969. 


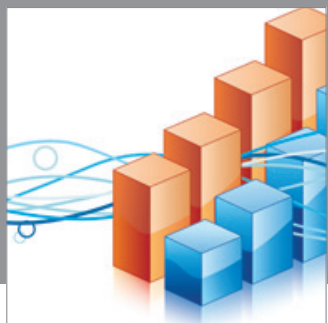

Advances in

Operations Research

mansans

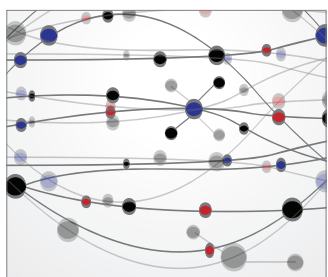

The Scientific World Journal
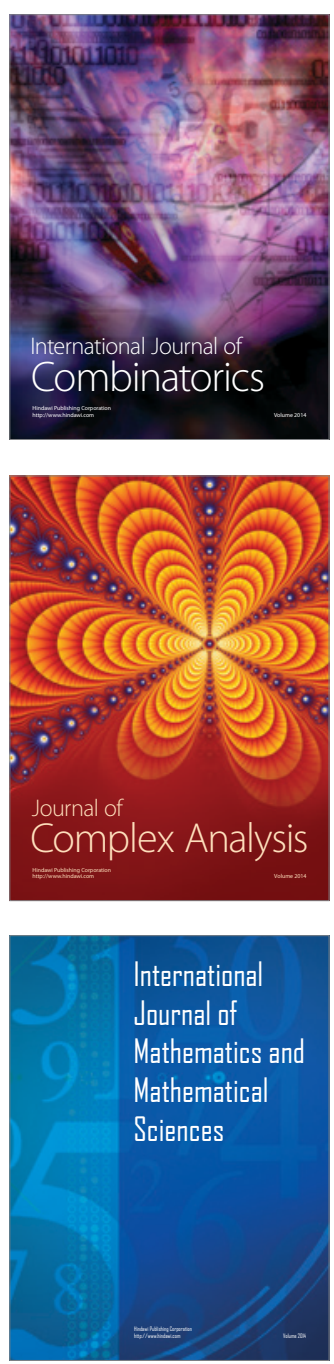
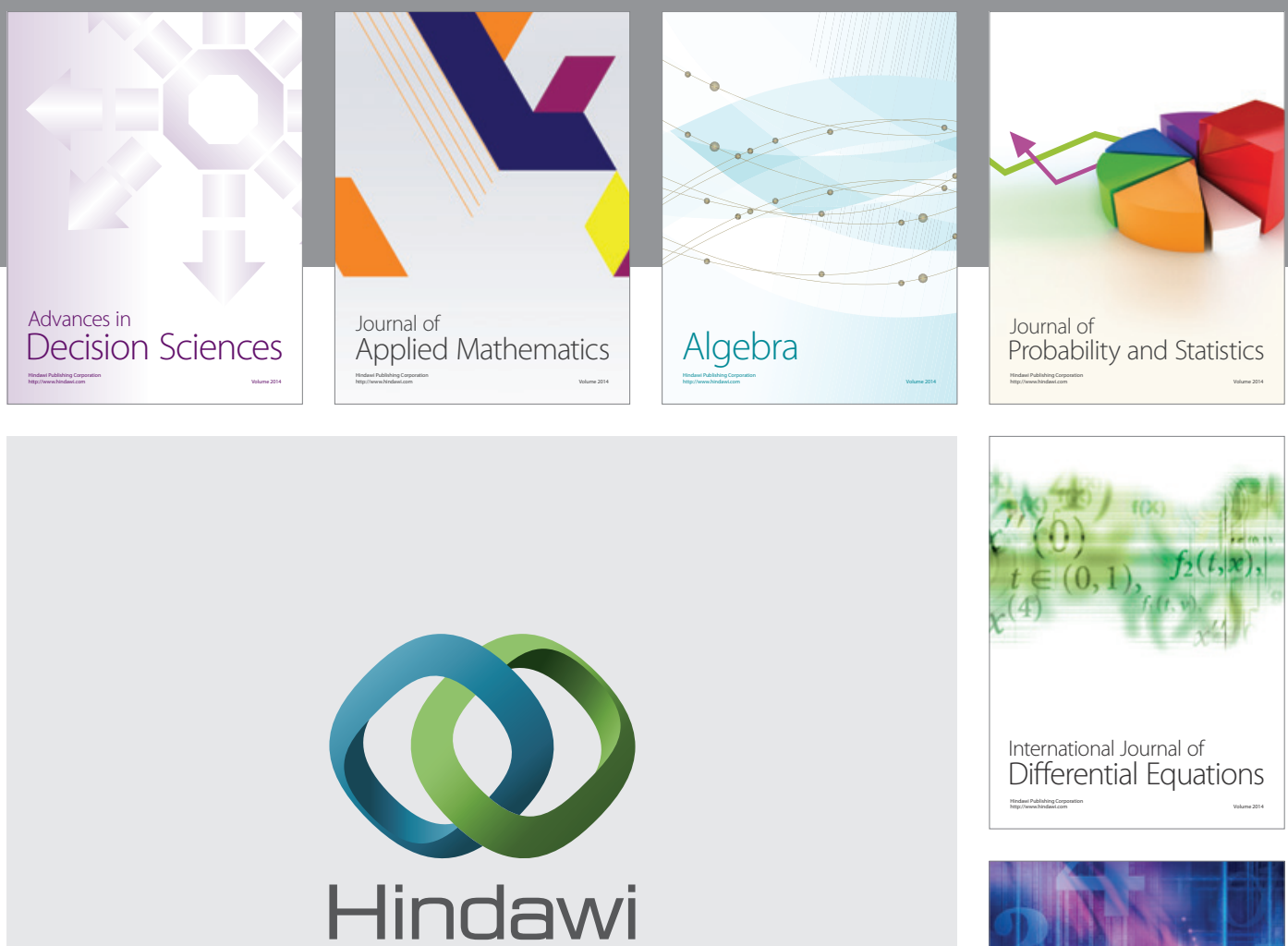

Submit your manuscripts at http://www.hindawi.com
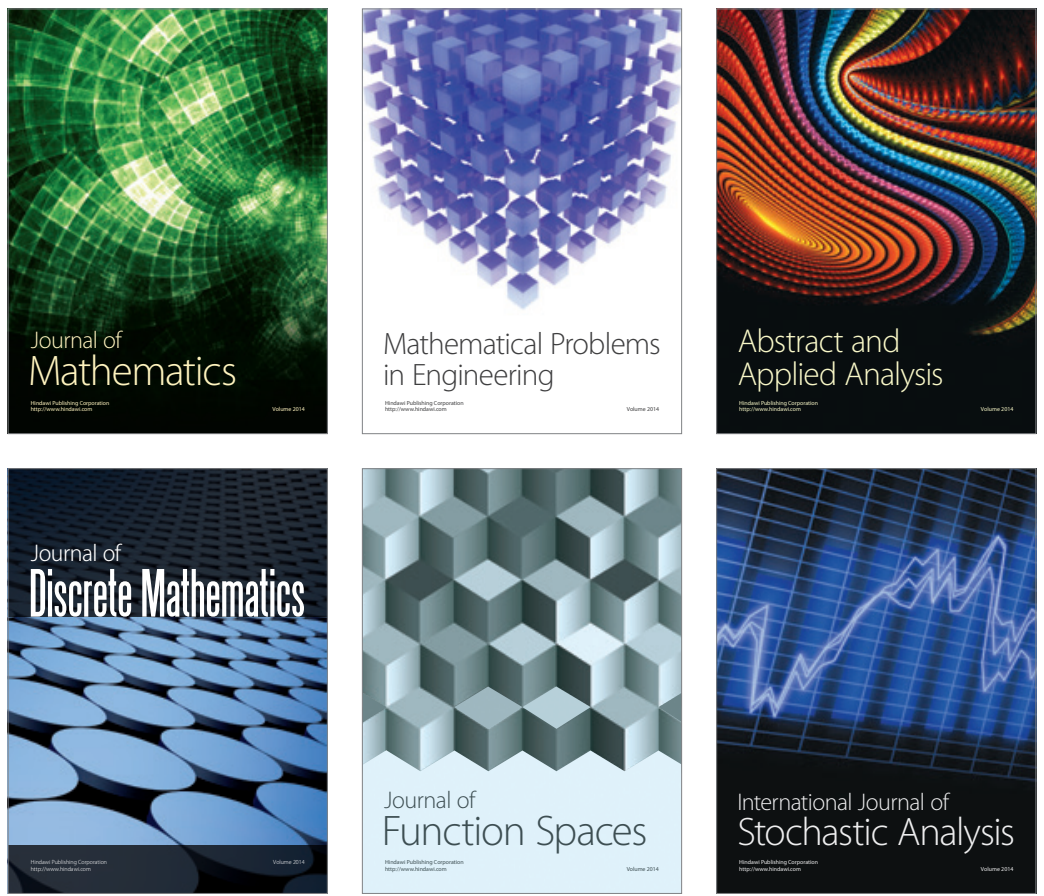

Journal of

Function Spaces

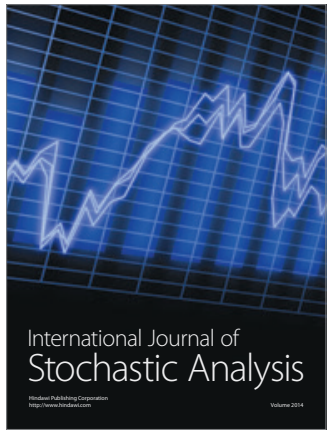

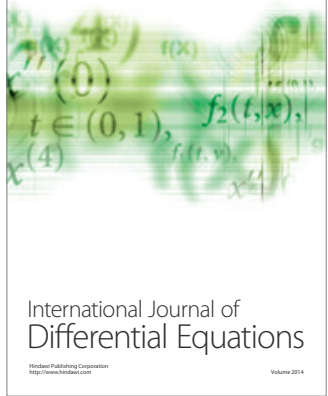
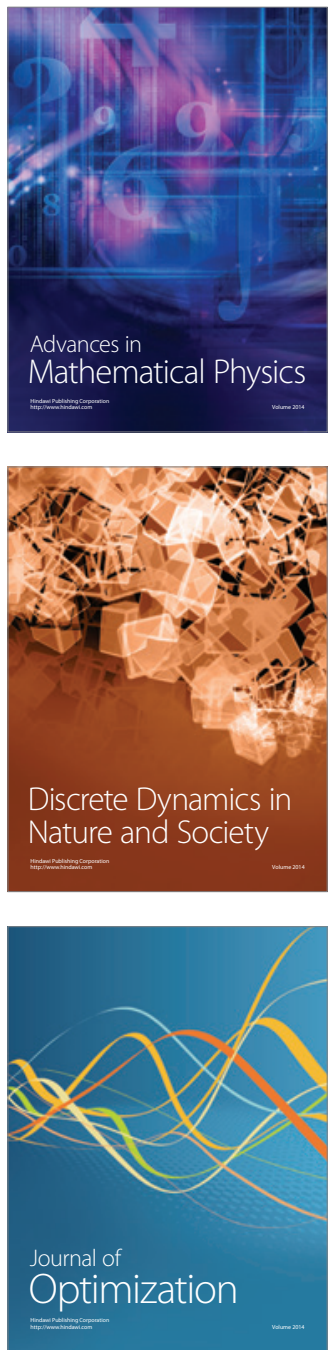